\title{
INVESTIGATION OF QUANTUM DOT STRUCTURES GROWN BY MOCVD IN InAs/GaAs SYSTEM
}

\author{
J. JASIŃSKI, R. BożeK, R. STĘPNIEWSKI \\ Institute of Experimental Physics, Warsaw University \\ Hoża 69, 00-681 Warsaw, Poland
}

\section{AND J. KozUBowski}

Faculty of Materials Science and Engineering, Warsaw University of Technology Narbutta 85, 02-524 Warsaw, Poland

\begin{abstract}
Layers of InAs quantum dots grown on [100] GaAs substrates were characterised by photoluminescence and investigated by transmission electron microscopy. Two types of InAs islands were observed in these layers. The islands of the first type had mainly a form of big, elongated pyramids. Most of them were found to be dislocated. On the other hand, the islands of the second type were real self-assembled, coherent quantum dots giving rise to a. characteristic photoluminescence band.
\end{abstract}

PACS numbers: $68.55 .-\mathrm{a}, 61.16 . \mathrm{Bg}$

In the last few years, the so-called quantum dots (QDs) known also as artificial atoms have attracted a lot of scientific interest. It has happened mostly because QDs open a lot of new possibilities for fundamental research and additionally, they can be applied in light-emitting devices $[1,2]$. There are a few different ways of obtaining QDs but probably the most interesting one makes the use of Stranski-Krastanov mode during epitaxial growth of semiconducting layer. Self-assembled QDs obtained in this way have been reported for many layer-substrate lattice-mismatched systems. InAs on GaAs is a classical example of such system. There have been many reports of self-assembled QDs obtained in this system. However, these dots were mostly grown by molecular beam epitaxy $[3,4]$ and there have been only a few reports on InAs QDs grown on GaAs by metal organic chemical vapour deposition (MOCVD). Therefore, in this paper we want to present our results of studies carried out on such QDs. We will mostly concentrate on morphology aspects and refer them to photoluminescence $(\mathrm{PL})$ results.

The layers of QDs used for this investigation were grown by MOCVD on [100] GaAs substrates. Different growth conditions, i.e., growth temperature and III/V 
ratio were applied. In most cases, the layers were capped with GaAs. Transmission electron microscopy (TEM) was performed on many of such samples. Mechanical thinning and dimpling followed by chemical etching or ion milling were applied for preparation of plain-view and cross-sectional specimens, respectively. Besides, the capped layers samples were characterised by PL.

The PL measurements of such samples revealed a characteristic emission band at energy in the range of about $1.0 \div 1.2 \mathrm{eV}$. In the literature such band is commonly attributed to radiative recombination within a large ensemble of slightly different QDs. A complex structure of this band - two or even more peaks - was often observed in our samples.
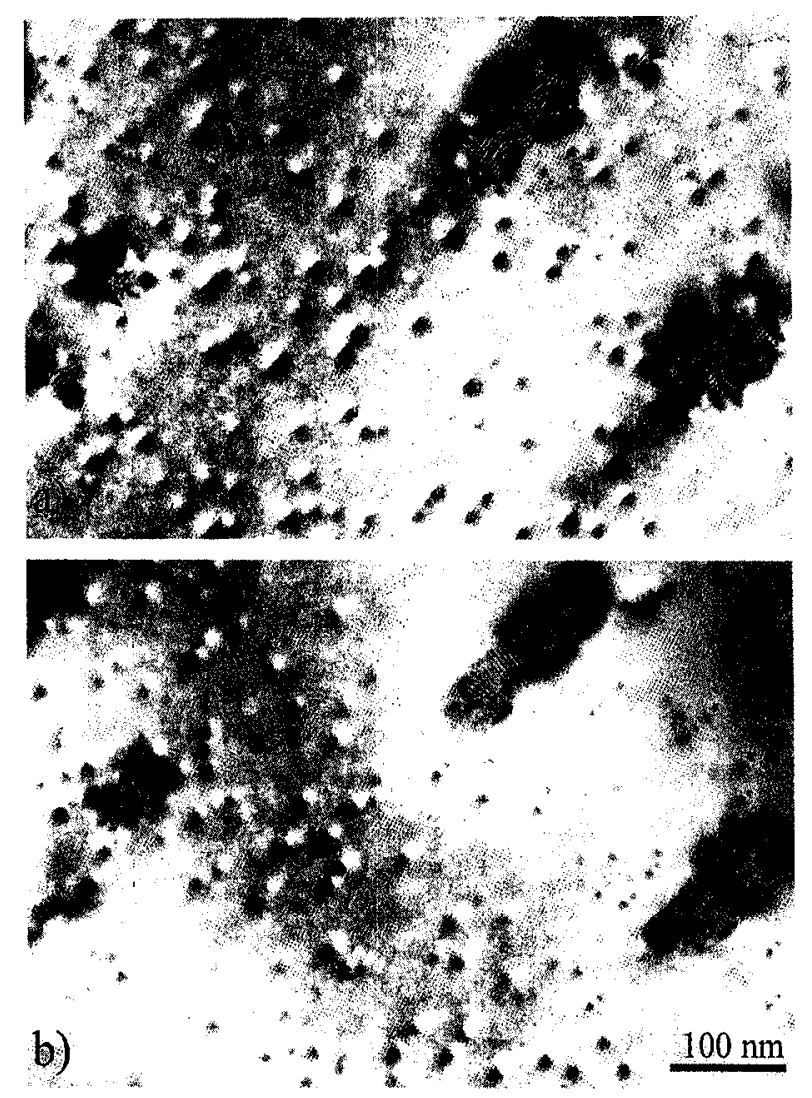

Fig. 1. Plain-view bright field TEM images of typical InAs layer covered with GaAs. A few big elongated InAs islands and many of self-assembled InAs QDs are visible. A careful comparison of both images ( $a$ and $b$ ), which were taken from the same specimen area but with different diffraction $g$-vectors (both $g$-vectors were of the [011]-type but perpendicular to each other), indicates a slight elongation of self-assembled dots. The direction of the elongation is the same as the direction of the big islands elongation. 
High resolution electron microscopy (HREM) studies of cross-sectional specimens revealed the presence of about 2-monolayers-thick InAs wetting layer. This layer was covered by two types of isolated InAs islands. They are visible on plain-view micrographs in Fig. 1. The islands of the first type were large with a broad size distribution. Their in-plane size changed in the range of about $20 \div 100 \mathrm{~nm}$. Most of them had pyramidal shapes, however, there were some islands, mainly in uncovered layers, with lens shapes. Almost all of these big islands had an elongated, nearly rectangular base. The elongation of all islands had the same [011]-type direction. The side walls of the pyramidal islands were formed on the (111)-type planes. The cross-sectional HREM image of such island is shown

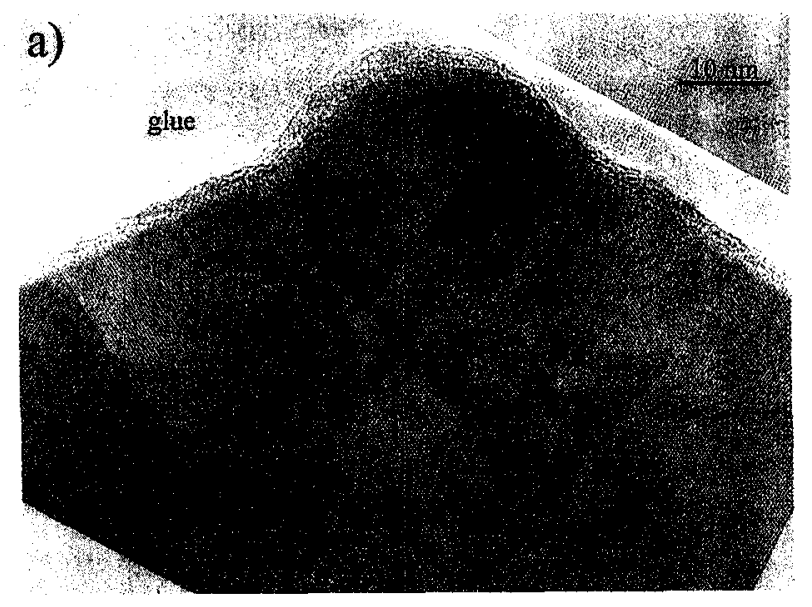

b)

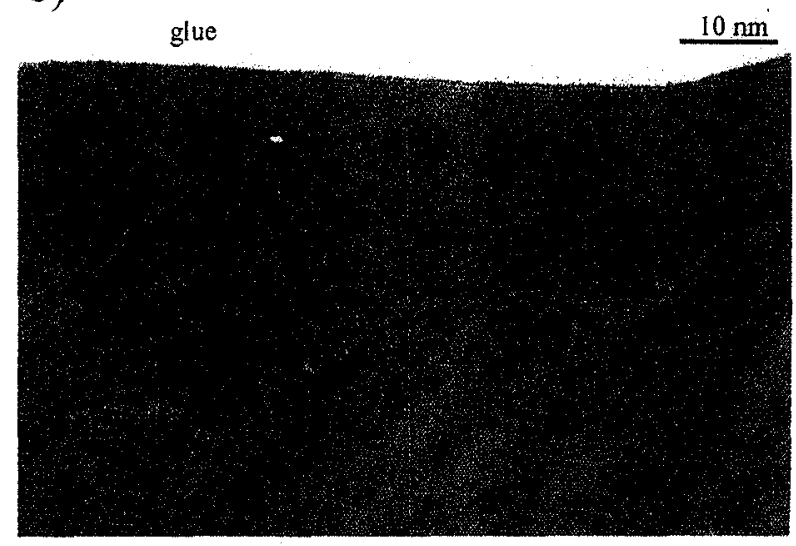

Fig. 2. Cross-sectional HREM images of typical InAs layer covered with GaAs. A big pyramidal island (a) and four self-assembled QDs (b) are visible. An array of misfit dislocation is indicated in the upper image. Strain field (dark contrast) around dots and trace of wetting layer (light line) can be seen in the lower image. 
in Fig. 2a. Microscopic studies showed that most of these big islands were dislocated. A regular array of misfit dislocations located at the interface between GaAs substrate and island base was often visible in HREM image of such island (see Fig. 2a). Dislocation density indicated not complete strain relaxation. This was in agreement with the results obtained from selected area diffraction patterns taken from the areas containing one of such islands. Thie selected area diffraction indicated also a slight strain difference within such islands for two perpendicular directions - parallel and normal to the direction of the island elongation. TEM studies showed additionally that the top surface of GaAs capping layer was not uniformly flat but that there were hills on the surface above such islands. Moreover, threading dislocations, generated at the interface between the islands and capping layer, were observed.

The islands of the second type were what one would like to call QDs. They were rather small and had a rather narrow size distribution. For example, for the samples grown in $470^{\circ} \mathrm{C}$ dots the average in-plane size was about $10 \pm 2 \mathrm{~nm}$. The density of these dots depended on the growth conditions and the highest obtained density was about $5 \times 10^{10} \mathrm{~cm}^{-2}$. All of these dots were nearly fully symmetrical in the layer plane. However, a comparison of two bright field TEM images obtained for two perpendicular $\boldsymbol{g}$ vectors, $\boldsymbol{g}_{(011)}$ (Fig. 1a) and $\boldsymbol{g}_{(01-1)}$ (Fig. 1b), indicated a slight dot elongation in the same direction as the elongation of the first-type islands. The same [011]-type direction for dots and big islands elongation could be associated with the direction of orbitals in the last atomic layer of [100] GaAs substrate. The cross-sectional HREM image of a few dots is shown in Fig. 2b. The analysis of similar images indicated that dots had lens shapes with a height to the in-plane size ratio in the range of about $0.14 \div 0.17$. Furthermore, the HREM images suggested that the strain field around such dots extended into GaAs at

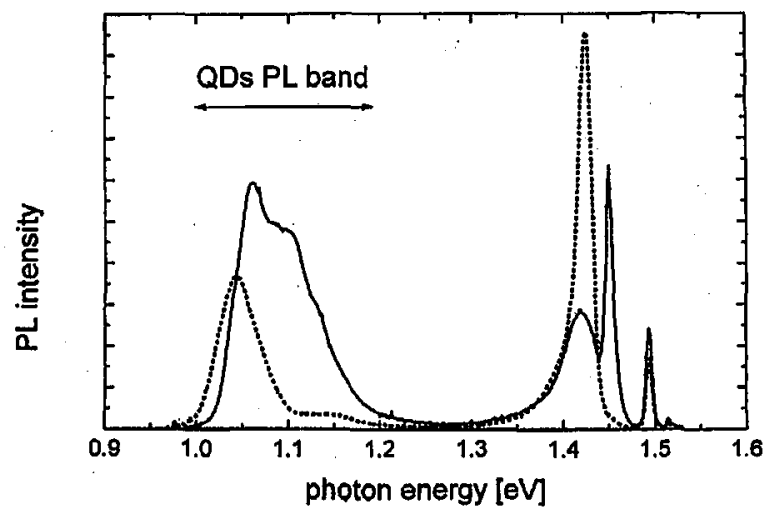

Fig. 3. $\mathrm{PL}$ intensity (in arb. units) of samples grown at $500^{\circ} \mathrm{C}$ (solid line) and $530^{\circ} \mathrm{C}$ (dotted line). Density and average in-plane size of self-assembled dots in these samples deduced from TEM were about: $3 \times 10^{10} \mathrm{~cm}^{-2}, 10.5 \pm 1.5 \mathrm{~nm}$ and $1 \times 10^{10} \mathrm{~cm}^{-2}, 15 \pm 2 \mathrm{~nm}$, respectively. One can notice a shift of characteristic PL band towards the lower energies with an increase in dot size. 
a distance of about 10 layers. Above this distance GaAs layers were found to be flat. The uniform shapes and a rather narrow size distribution of these second-type islands indicated that they really were self-assembled QDs. This was also confirmed by the correlation between dot density and intensity of the characteristic PL band. Besides, the correlation between the average dot size and the emission energy was also observed (Fig. 3).

To summarise, the results of PL and TEM studies performed on the samples containing InAs QDs grown on [100] GaAs were presented. Two types of InAs islands were observed; the big, dislocated islands and real QDs associated with the characteristic PL band. It was found that higher growth temperature resulted in lower QDs density, bigger average dot size and lower energy of the QDs emission measured in PL spectrum.

J. J. wants to thank Dr. Peter Werner for making possible carrying out the cross-sectional TEM studies in Max Planck Institute for Microstructure Physics in Halle, Germany. This work was partially supported by the Committee for Scientific Research grant No. PBZ28.11/P4 and 8T11B 003312.

\section{References}

[1] N. Kirsteadter, N.N. Ledenstenov, M. Grudmann, D. Bimberg, V.M. Ustinov, S.S. Ruvimov, M.V. Maximov, P.S. Kopev, Zh.I. Alferov, U. Richter, P. Werner, U. Gosele, J. Heydenreich, Electron. Lett. 30, 1416 (1994).

[2] H. Shoji, Y. Nakata, K. Mukai, Y. Sugiyama, M. Sugawara, N. Yokoyama, H. Ishikawa, Appl. Phys. Lett. 71, 193 (1997).

[3] D. Leonard, M. Krishnamurthy, C.M. Reaves, S.P. DenBaars, P.M. Petroff, Appl. Phys. Lett. 63, 3203 (1993).

[4] N.N. Ledenstenov, M. Grudmann, N. Kirsteadter, O. Schmidt, R. Heitz, J. Bohrer, D. Bimberg, V.M. Ustinov, V.A. Schukin, A.Yu. Egorov, A.E. Zhukov, S. Zaitsev, P.S. Kopev, Zh.I. Alferov, S.S. Ruvimov, P. Werner, U. Gosele, J. Heydenreich, Solid State Electron. 40, 785 (1996). 